\title{
Dual-Priming Isothermal Amplification (DAMP) for Highly Sensitive and Specific Molecular Detection with Ultralow Nonspecific Signals
}

\author{
Xiong Ding ${ }^{\mathrm{a}}$, Zhiheng $\mathrm{Xu}^{\mathrm{a}}$, Kun Yin ${ }^{\mathrm{a}}$, Maroun Sfeir ${ }^{\mathrm{b}}$, Changchun Liu ${ }^{\mathrm{a}},{ }^{*}$ \\ a Department of Biomedical Engineering, University of Connecticut Health Center, Farmington, \\ Connecticut 06030, USA \\ b Department of Pathology and Laboratory Medicine, University of Connecticut Health Center, \\ Farmington, Connecticut 06030, USA
}

\section{Abstract}

\begin{abstract}
Nucleic acid amplification tests have been widely used in clinical diagnostics, food safety monitoring, and molecular biology. Loop-mediated isothermal amplification (LAMP) is a prevailing nucleic acid isothermal amplification method. It has become a powerful alternative to conventional polymerase chain reaction (PCR) for pathogen detection because of its simplicity, rapidity, and high sensitivity. However, the current LAMP methods, especially the LAMP with two loop primers, suffer from undesired nonspecific amplification with strong background signals due to the increasing target sites. This nonspecific amplification substantially reduced the reliability of the LAMP and limited its applications in clinical diagnostics. Here, we report a "dual-priming" ("self-priming" and "pairing-priming") isothermal amplification (DAMP) assay for rapid nucleic acid detection with ultralow nonspecific signals. This method takes advantage of the "dualpriming" strand extension strategy by adding two pairing-competition primers and designing unique inner primers, enabling highly sensitive and specific molecular detection. As an application demonstration, DAMP assay was used to detect HIV-1 DNA/ RNA and Escherichia coli DNA, showing equal or better sensitivity with shorter detection time compared to conventional LAMP and PCR methods. More importantly, the DAMP assay showed ultralow background signals without false positive signals even after 2-hour incubation. Such simple, reliable, sensitive, and specific DAMP assay can be well suitable for rapid nucleic acid detection as point of care testing, particularly in the resource-limited settings.
\end{abstract}

\section{Graphical Abstract}

\footnotetext{
*Corresponding Author, Phone: (860)-679-2565. chaliu@uchc.edu. Author Contributions

The manuscript was written through contributions of all authors. ASSOCIATED CONTENT

Supporting Information

The Supporting Information is available free of charge on the ACS Publications website.

List of sequence information of the primers (Table S1) and targets and the supplementary figures (Figure S1-S20) (PDF).
} 


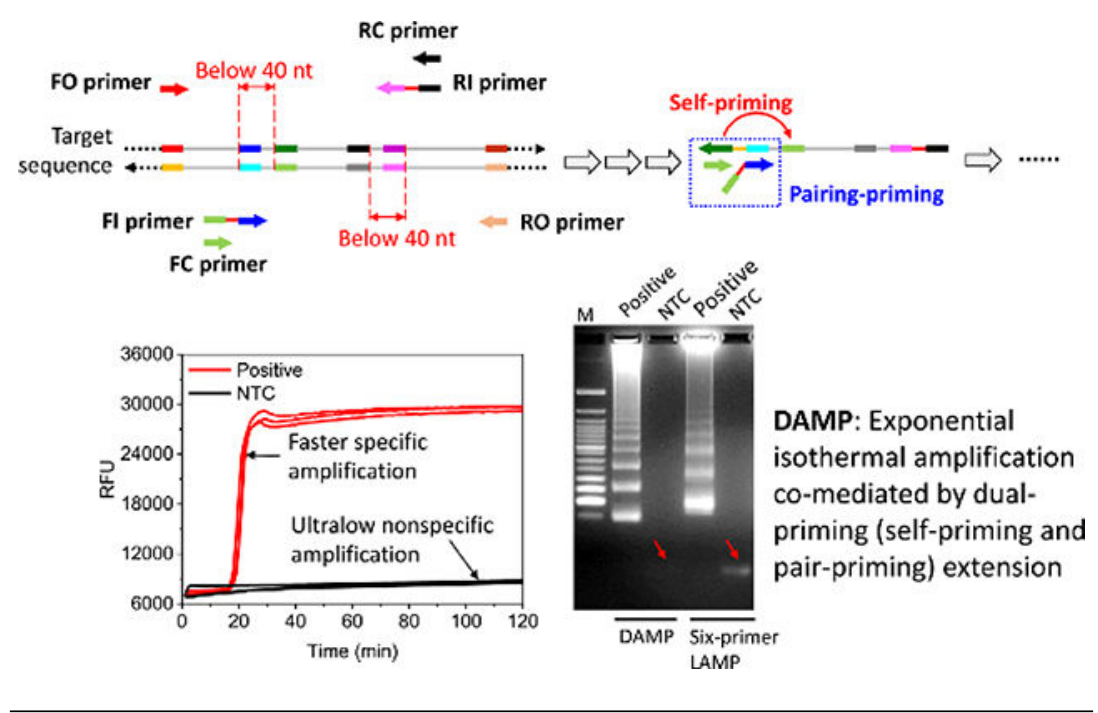

\section{INTRODUCATION}

Nucleic acid amplification tests (NAATs) have been used in many fields including clinical molecular diagnostics, food safety monitoring, gene expression analysis, and fundamental molecular biology. ${ }^{1-4}$ Polymerase chain reaction (PCR) is the most common approach for DNA amplification and pathogen identification in clinical microbiology laboratories.

However, PCR typically requires expensive equipment and well-trained personnel, which is not suitable for point of care diagnostics and/ or testing in resource-limited settings. For the past few decades, with the rapid development of enzyme engineering and molecular biology, NAATs have been significantly simplified with emerging isothermal amplification technologies $^{5}$, such as loop-mediated isothermal amplification (LAMP) ${ }^{6}$, nucleic acid sequence based amplification (NASBA) ${ }^{7}$, recombinase polymerase amplification (RPA) ${ }^{8}$, and helicase dependent amplification (HDA) ${ }^{9}$. Among these, LAMP method is one of the most widely used isothermal amplification assays for pathogen detection due to its simplicity, rapidity, high sensitivity and cost-effectiveness. ${ }^{10,11}$

The earliest LAMP technology proposed by Notomi et al. ${ }^{6}$ used four primers to recognize six distinct regions of target DNA, termed "four-primer LAMP". However, the four-primer LAMP assay is too slow for many clinical diagnostic applications. To improve amplification efficiency and shorten detection time, several LAMP variants have been developed by adding loop primers ${ }^{12}$, stem primers ${ }^{13}$ and swarm primers ${ }^{14}$. Of these variants, LAMP with two loop primers (termed "six-primer LAMP") has prevailed following the addition of loop primers which greatly improved amplification efficiency and reduced testing time. ${ }^{12}$

Although stem primers were developed to accelerate the amplification reaction ${ }^{13}$ they added a challenge to the primer design of the six-primer LAMP since six primers need to recognize eight distinct sites of the target DNA. To reduce the number of target sites, swarm primers were proposed to be added to the LAMP. ${ }^{14}$ Unfortunately, the amplification efficiency of the LAMP with swarm primers only is lower than that of the LAMP with loop or stem primers. To ensure rapid amplification, loop/stem primers were added to the LAMP with swarm primers, which increases the total number of target sites up to ten. ${ }^{14}$ However, the nature of 
increasing target sites in the LAMP assays is prone to form primer dimers and leads to undesired nonspecific amplification, which limits the detection reliability and specificity in clinical diagnostic applications. Furthermore, all these LAMP methods mainly rely on the inner primers to initiate a "self-priming" strand extension during the isothermal amplification with less ability to simultaneously generate multiple basic structures for cycling amplification.

Herein, we report a "dual-priming" isothermal amplification method (including "selfpriming" and "pairing-priming" strand extension, termed "DAMP") for highly sensitive and specific nucleic acid detection with ultralow nonspecific signals. To enable a fairly competitive strand extension between "self-priming" and "pairing-priming", we incorporated two strategies into our DAMP assay: i) we modified the inner primer design by reducing the distance of its two target sites below 40 nucleotides (nt) (typically 40-60 nt for conventional LAMP method ${ }^{15}$ ), and ii) we added two pairing-competition primers to recognize the sites at the free $3^{\prime}$-end parts of the basic structures. The pairing-competition primers compete with the "self-priming" extension to enable efficient "pairing-priming" extension, thereby generating multiple basic structures simultaneously (e.g., the initial amplicons generated by the inner primers) for cycling amplification. Our study demonstrated that the "dual-priming" strand extension is critical to achieve a highly sensitive and reliable DAMP assay. The performance of our DAMP assay was evaluated by detecting HIV-1 DNA/RNA and Escherichia coli (E. coli) DNA. Our experiment showed that DAMP assay had equal or better sensitivity with faster amplification speed compared to conventional LAMP and PCR assays. Most importantly, the DAMP assay shows ultralow nonspecific background signals even after two-hour incubation. Therefore, our DAMP assay is a simple, versatile, sensitive and specific method for rapid nucleic acid detection.

\section{EXPERIMENTAL SECTION}

\section{Materials and Reagents}

Fluorescent dye EvaGreen ${ }^{\circledR}(20 \times$ in water) was purchased from Biotium (Fremont, CA). Agarose powder, $50 \times$ TAE (Tris/Acetic Acid/EDTA) Buffer, and SsoAdvanced ${ }^{\mathrm{TM}}$ Universal $\mathrm{SYBR}^{\circledR}$ Green PCR Supermix were purchased from Bio-Rad Laboratories (Hercules, CA). Deoxynucleotide (dNTP) solution mix (10 mM of each), $\mathrm{Mg}_{2} \mathrm{SO}_{4}(100 \mathrm{mM})$, Bacillus stearothermophilus (Bst) 2.0 WarmStart ${ }^{\circledR}$ DNA polymerase $(8000 \mathrm{U} / \mathrm{mL})$, WarmStart ${ }^{\circledR}$ RTx Reverse Transcriptase (15,000 units/mL), extreme thermostable single-stranded DNA binding protein (ET SSB, $500 \mu \mathrm{g} / \mathrm{mL}$ ), $10 \times$ Isothermal Amplification Buffer $(200 \mathrm{mM}$ Tris$\mathrm{HCl}, 500 \mathrm{mM} \mathrm{KCl}, 100 \mathrm{mM}\left(\mathrm{NH}_{4}\right)_{2} \mathrm{SO}_{4}, 20 \mathrm{mM} \mathrm{MgSO}_{4}, 1.0 \%$ Tween ${ }^{\circledR} 20$ and $\mathrm{pH} 8.8$ at $25^{\circ} \mathrm{C}$ ), and 10 ThermoPol Reaction Buffer (200 mM Tris- $\mathrm{HCl}, 100 \mathrm{mM} \mathrm{KCl}, 100 \mathrm{mM}$ $\left(\mathrm{NH}_{4}\right)_{2} \mathrm{SO}_{4}, 20 \mathrm{mM} \mathrm{MgSO}_{4}$ and $1 \%$ Triton $\mathrm{X}-100, \mathrm{pH} 8.8$ at $25^{\circ} \mathrm{C}$ ) were all purchased from New England BioLabs (Ipswich, MA). RNeasy Mini Kit for RNA extraction was purchased from QIAGEN (Frederick, MD). AcroMetrix ${ }^{\mathrm{TM}}$ HIV-1 Controls was purchased from Thermo Fisher Scientific (Waltham, MA). Oligonucleotides (primers) and the pUCIDT (Amp) plasmid containing 300-bp HIV-1 p24 gene or 300-bp E. coli B malB gene sequence were ordered from Integrated DNA Technologies (Coralville, IA). 


\section{DAMP and RT-DAMP assays}

For the DAMP assay, a total of six primers were used to recognize six different target sites (Figure 1A), including the forward and reverse outer primers (FO and RO primers), the forward and reverse inner primers (FI and RI primers), as well as forward and reverse pairing-competition primers (FC and RC primers). The DAMP primers were manually designed using the OligoAnalyzer Tool and the PrimerExplorer (http://primerexplorer.jp/e/) according to the principle of primer design shown in Figure S2. Alternatively, we also developed an online primer design platform (https://github.com/xuzhiheng001/DAMPDesign) to assist DAMP primer design. The optimal DAMP reaction system contained 0.2 $\mu \mathrm{M}$ each of FO and RO primers, $1.6 \mu \mathrm{M}$ each of FI and RI primers, $1.6 \mu \mathrm{M}$ each of FC and RC primers, $1 \times$ EvaGreen $^{\circledR}, 0.2 \mathrm{M}$ betaine, $1 \times$ Isothermal Amplification Buffer $(20 \mathrm{mM}$ Tris- $\mathrm{HCl}, 50 \mathrm{mM} \mathrm{KCl}, 10 \mathrm{mM}\left(\mathrm{NH}_{4}\right)_{2} \mathrm{SO}_{4}, 2 \mathrm{mM} \mathrm{MgSO}_{4}$, and $0.1 \%$ Tween $\left.{ }^{\circledR} 20\right), 1.2 \mathrm{U} / \mu \mathrm{L}$ Bst 2.0 WarmStart ${ }^{\circledR}$ DNA polymerase, $1.6 \mathrm{mM}$ of each dNTP, $4 \mathrm{mM} \mathrm{MgSO}_{4}$, and $1.0 \mu \mathrm{L}$ of plasmid template solution. Before adding the DNA templates for isothermal amplification, the plasmid template solutions were denatured first by heating to $95^{\circ} \mathrm{C}$ for $5 \mathrm{~min}$ and then chilling on ice for $5 \mathrm{~min}$. The optimal RT-DAMP reaction system was the same as the optimal DAMP reaction system, except for the addition of $0.3 \mathrm{U} / \mu \mathrm{L}$ WarmStart ${ }^{\circledR} \mathrm{RTx}$ Reverse Transcriptase and $2.5 \mathrm{ng} / \mu \mathrm{L}$ Extreme Thermostable Single-stranded DNA Binding Protein (ET SSB). The sequences of primers and targets are shown in Table S1.

\section{LAMP and RT-LAMP assays}

The LAMP primers were all designed using the online PrimerExplorer (http:// primerexplorer.jp/e/). The optimal LAMP reaction protocol was developed according to the guideline of New England BioLabs (https://www.neb.com/protocols/2014/11/21/typicallamp-protocol-m0275), which consisted of $0.2 \mu \mathrm{M}$ each of F3 and B3 primers, $1.6 \mu \mathrm{M}$ each of FIP and BIP primers, $0.4 \mu \mathrm{M}$ each of LoopF and LoopB primers, $1 \times$ EvaGreen $^{\circledR}, 1 \times$ Isothermal Amplification Buffer, 0.32 U/ $\mu \mathrm{L}$ Bst 2.0 WarmStart ${ }^{\circledR}$ DNA polymerase, $1.4 \mathrm{mM}$ of each dNTP, $6 \mathrm{mM} \mathrm{MgSO}_{4}$, and $1.0 \mu \mathrm{L}$ of plasmid template solution. The RT-LAMP reaction system was similar to the the LAMP reaction system but supplemented with $0.3 \mathrm{U} /$ $\mu \mathrm{L}$ WarmStart ${ }^{\circledR}$ RTx Reverse Transcriptase and $2.5 \mathrm{ng} / \mu \mathrm{L}$ ET SSB. The reaction systems for LAMP with swarm primers were the same as what the literature reported previously. ${ }^{14}$ For the LAMP assay of E. coli $\mathrm{B}$ malB gene sequence, the primer sequences and LAMP assay protocol were similar to previous study ${ }^{16}$.

\section{PCR assay}

SsoAdvanced ${ }^{\mathrm{TM}}$ Universal SYBR ${ }^{\circledR}$ Green Supermix from the Bio-Rad Laboratories was used for the PCR assay. According to the instruction manual, the PCR reaction system contained $1 \times$ Supermix, $400 \mathrm{nM}$ each of primers, and $1.0 \mu \mathrm{L}$ of plasmid template solution. The thermal cycling protocol included $2.5 \mathrm{~min}$ at $98{ }^{\circ} \mathrm{C}$ for initial denaturation, 35 cycles of $15 \mathrm{~s}$ at $95^{\circ} \mathrm{C}$ for denaturation and $30 \mathrm{~s}$ at $60^{\circ} \mathrm{C}$ for annealing and extension, and the meltcurve analysis (from $65{ }^{\circ} \mathrm{C}$ to $95{ }^{\circ} \mathrm{C}$ with $0.5{ }^{\circ} \mathrm{C}$ increment). The sequences of primers and targets are shown in Table S1. 


\section{Reaction condition and product analysis}

The real-time fluorescence detection of DAMP and LAMP was performed in the CFX96 Touch ${ }^{\mathrm{TM}}$ Real-Time PCR Detection System (Bio-Rad) by incubating the reactions at $60{ }^{\circ} \mathrm{C}$ for $60 \mathrm{~min}$ or $120 \mathrm{~min}$, followed by heating at $80^{\circ} \mathrm{C}$ for $20 \mathrm{~min}$. The threshold-time of our DAMP and LAMP assay was defined as the maximum of the second derivative from the fluorescence curve. The data analysis was accomplished by using the Prism 8 (GraphPad Software, GSL Biotech LLC, San Diego, CA). The amplified products were analyzed through the electrophoresis in a $3.0 \%$ agarose gel $(1 \times$ TAE $)$ stained with $1 \times$ EvaGreen $^{\circledR}$.

\section{RESULTS AND DISCUSSION}

\section{Principle of the DAMP assay}

Figure 1 illustrates the principle of the DAMP assay. As shown in Figure 1A, the DAMP assay employs six primers: i) two outer primers (FO and RO), ii) two inner primers (FI and $\mathrm{RI}$ ), and iii) two pairing-competition primers (FC and $\mathrm{RC}$ ). Unlike conventional six-primer LAMP assay targeting eight distinct sites (Figure S1), the six primers of the DAMP recognize six distinct sites of the target DNA sequences which contain: i) F1, R2, R3 sites on the forward target sequence, and ii) F3c, F2c, R1c sites on the reverse sequence. F and R stand for the "forward" and "reverse" directions, respectively. The sites with lowercase "c" represent "complementary" sites. Compared to conventional LAMP primers design (Figure S1A), the DAMP method has two distinct features (Figure 1A): i) each inner primer is designed to recognize two target sites with the distance of below $40 \mathrm{nt}$, which ensures efficient "dual-priming" extension. In addition, a four-thymine oligonucleotide spacer (TTTT spacer) is introduced into the inner primers. However, in the LAMP method, the distance between two target sites of the inner primers is recommended to be 40-60 nt to ensure efficient "self-priming" extension according to the LAMP primer design guide, ${ }^{15}$ and ii) two pairing-competition primers are added to initiate the "dual-priming" extension and accelerate the amplification. Interestingly, the addition of the pairing-competition primers does not increase the total number of target sites because their sequences are the same as the $5^{\prime}$-parts of the inner primers (FI/RI). The length and melting temperature $\left(T_{m}\right)$ for each site are summarized in Figure S2. To facilitate the primers design, we developed an online software by using Python (https://github.com/xuzhiheng001/DAMP-Design).

The DAMP assay typically contains two steps: i) basic structure producing step (Figure 1B) and ii) cycling amplification step (Figure 1C and S3). We used the reverse target sequence as an example to facilitate the explanation of the DAMP amplification mechanism. In the DAMP assay, DNA synthesis is initiated by DNA polymerase when the F2 region of FI primer anneals to the F2c site and the FO primer anneals to the F3c site. Due to the stranddisplacement activity of the DNA polymerase, the strand elongated from FI primer is displaced and released by the FO primer extension. Then, the R2 and R3 sites in the released strand are recognized by the RI and RO primers, respectively. Afterwards, the extended strand by RI primer is displaced by the elongated strand from the RO primer. The released strand forms basic structure (termed "Basic Structure 1" in Figure 1B) for downstream cycling amplification. Since the RI primer contains two target sites with a distance of less than $40 \mathrm{nt}$, the "self-priming" strand extension capability in Basic Structure 1 is reduced 
compared to the dumb-bell structure in the conventional LAMP method (Figure S1). $6,17,18$

Furthermore, due to the addition of pairing-competition primers, the "self-priming" extension (Item 1 in the black box in Figure 1C) will fully compete with three "pairingpriming" extension events: i) the first "pairing-priming" extension is initiated by the annealing of the F2 site in the FI primer to the F2c site (Item 2 in the box), ii) the second one results from the annealing competition of FC primer to the F1 site (Item 3 in the box), and iii) the third one is the concurrence of two aforementioned events (Item 4 in the box). The "self-priming" extension is able to produce double-stranded DNA (dsDNA) fragments with a closed loop which can be recognized by the FI primer. Then, the "self-priming" and "pairing-priming" strand extensions take place at their $3^{\prime}$-end parts again, and the amplification proceeds to Pathway 1. Unlike the "self-priming" extension, the "pairingpriming" extension can simultaneously generate multiple basic structures for cycling amplification, including duplex basic structure and complementary basic structure (Basic Structure 2 in Figure 1C). Similarly, "self-priming" and "pairing-priming" extensions occur at both ends of the duplex basic structure, then stepping into Pathways 1 and 2. Whereas, for Basic Structure 2, the "dual-priming" extension occurs only at the 3 '-end part, and then moves into the Pathway 2. In the Pathways 1 and 2, owing to the co-mediation effect of "self-priming" and "pairing-priming" extensions, the Basic Structure 1 and 2 are generated again to recycle the amplification. Details on these two pathways are shown in Figure S3.

\section{Dual-priming extension effect of the DAMP assay}

To investigate the "dual-priming" extension mechanism of the DAMP assay, we designed and tested three sets of DAMP primers for HIV-1 p24 gene detection, which contained the same outer and pairing-competition primers but different inner primers with various distances between two target sites (from $5^{\prime}$-end of F2 to $5^{\prime}$-end of F1 and from $3^{\prime}$-end of R1 to $5^{\prime}$-end of R2) in the target sequences (Figure 2). We first designed DAMP inner primers by setting the distance of their two target sites to be 55 and $56 \mathrm{nt}$ (Figure 2A, Left) and performed real-time DAMP. As shown in Figure 2A, Right, it showed low amplification efficiency and obvious nonspecific amplification with elevated baselines. The low amplification efficiency may be attributed to dominant "self-priming" strand extension like the conventional four-primer LAMP. The undesirable nonspecific amplification signals could likely be caused by the formation of primer dimer (Figure S4A). After reducing the distance to less than $40 \mathrm{nt}$ (e.g., $30 \mathrm{nt}$ ), both "self-priming" and "pairing-priming" strand extensions were fully initiated and fairly competed with each other in the DAMP amplification. Such "dual-priming" effect produced more basic structures to mediate downstream cycling amplifications, enabling high efficiency DAMP amplification reaction with ultralow nonspecific signals (Figure 2B). However, upon further reducing the distance (e.g., $22 \mathrm{nt}$ ) (Figure 2C), the "self-priming" extension capability is dramatically suppressed, which influenced the formation of basic products and resulted in a low amplification efficiency. To further confirm this, their DAMP products were subjected to gel electrophoresis (Figure S4). Therefore, these results demonstrated that the optimization of the distance was necessary to design efficient primers for highly sensitive DAMP assay.

To further confirm that "dual-priming" extension is crucial in our DAMP assay, we also investigated the effect of single "self-priming" and single "pairing-priming" extension on 
isothermal amplification by fixing the distance of two target sites at $30 \mathrm{nt}$ for the inner primer design. First, we deleted respectively the F1c and R1 region of the FI and RI primers to only initiate single "pairing-priming" strand extension (Figure S5A, Left). As shown in Figure S5A, Right, the amplification efficiency of such single "pairing-priming" extension was very low. Next, we did not add the pairing-competition primers (FC/RC) to initiate single "self-priming" extension (Figure S5B, Left). As shown in Figure S5B, Right, single "self-priming" extension had lower amplification efficiency with increased detection time compared to the "dual-priming" extension (Figure S5C). Therefore, the results demonstrate that the "dual-priming" extension effect plays a critical role in achieving highly efficient and fast nucleic acid isothermal amplification in our DAMP assay.

\section{Optimization of the DAMP assay}

To achieve a highly sensitive detection of nucleic acids, we first optimized the DAMP reaction by investigating the effect of the concentration of various composites (e.g., DNA polymerase, dNTPs, betaine, and $\mathrm{MgSO}_{4}$ ). To investigate the DNA polymerase's effect, the concentration of DNA polymerase was increased from $0.16 \mathrm{U} / \mathrm{uL}$ to $1.2 \mathrm{U} / \mathrm{uL}$. As shown in Figure $3 \mathrm{~A}$, the higher the DNA polymerase concentrations was, the faster the amplification was as well. In our experiment, 1.2 U/ $\mu \mathrm{L}$ DNA polymerase was used as the optimal concentration because of its fastest speed. It should be mentioned that the increasing polymerase amount did not cause false positive signal in our DAMP assay (Figure S6) unlike the conventional LAMP assay (typically $0.32 \mathrm{U} / \mu \mathrm{L}$ polymerase). ${ }^{6,18}$ Then, we investigated the effect of dNTPs, betaine, and $\mathrm{MgSO}_{4}$ on the DAMP assay. During the nucleic acid amplification, the dNTPs are essential to build blocks of nucleic acid molecules. With the increase of dNTPs concentration, the threshold time became shorter (Figure 3B). To ensure highly specific isothermal amplification, $1.6 \mathrm{mM}$ dNTPs was chosen in our DAMP assay (Figure 3B and S7). Betaine is often used as an isostabilizing additive of isothermal amplification reactions to reduce dsDNA's $T_{m}$ and facilitate the strand separation. ${ }^{19-21}$ In our DAMP assay, $0.2 \mathrm{M}$ betaine was optimal because it provided the fastest amplification speed with high specificity (Figure 3C and Figure S8). Magnesium ions have been known to act as cofactor of DNA polymerase. ${ }^{22}$ Thus, various concentrations of magnesium ions were assessed in the DAMP assay. As shown in Figure 3D and S9, when using $4 \mathrm{mM} \mathrm{MgSO}_{4}$, DAMP amplification could be achieved within the shortest time and did not result in nonspecific amplification.

Next, the concentrations of primers were investigated and optimized. As shown in Figure 4A-C and Figure S10-12, the optimal concentrations of FO \& RO, FI \& RI, and FC \& RC were $0.2 \mu \mathrm{M}, 1.6 \mu \mathrm{M}$, and $1.6 \mu \mathrm{M}$, respectively. Thus, the optimized DAMP reaction solution contains $1.2 \mathrm{U} / \mu \mathrm{L}$ polymerase, $1.6 \mathrm{mM}$ each of dNTPs, $0.2 \mathrm{M}$ betaine, $4 \mathrm{mM}$ $\mathrm{MgSO}_{4}, 0.2 \mu \mathrm{M}$ FO \& RO, $1.6 \mu \mathrm{M}$ FI \& RI, and $1.6 \mu \mathrm{M}$ FC \& RC. With the optimized DAMP assay, we could rapidly detect HIV-1 DNA with ultralow nonspecific signal (Figure 4D).

\section{Nonspecific amplification evaluation of the DAMP assay}

To evaluate the ultralow nonspecific amplification of our DAMP assay, we designed and tested both six-primer LAMP and DAMP assay by targeting the same DNA sequence 
(Figure 5A, Left and 5B, Left). As shown in Figure 5A, Right, obviously ascended baselines were observed for NTC in the six-primer LAMP assay, which was caused by the formation of primer dimers. On the contrary, the DAMP assay had significantly lower initial background baseline signal (Figure 5B, Right). To further confirm that the rising baseline of the six-primer LAMP assay originated from the primer dimers, the amplification products of both DAMP and LAMP assays were subject to gel electrophoresis analysis. As shown in Figure 5C, nonspecific amplicon band was clearly observed in the NTC of the six-primer LAMP assay but not seen in the DAMP's NTC. In addition, melting curve analysis results further confirmed that the nonspecific amplification was observed only in the six-primer LAMP assay (Figure S13). Due to the ultralow nonspecific amplification, the endpoint fluorescence difference between positive and negative products of the DAMP assay was at least twice as high as that of the six-primer LAMP (Figure 5D). That is beneficial for many microfluidic-based point-of-care molecular detection. ${ }^{23,24}$ Compared to the six-primer LAMP, DAMP assay showed higher amplification yield (Figure 5D) and faster reaction speed (Figure S14). That could be possibly attributed to extra cycling amplifications resulting from multiple basic structures produced by "dual-priming" extension in the DAMP assay.

Furthermore, we compared the performance of our DAMP assay to other LAMP variants (Figure S15): i) four-primer LAMP (without two loop primers), ii) four-primer LAMP with two swarm primers, and iii) six-primer LAMP with the swarm primers. ${ }^{14}$ In order to ensure reliable comparison, the primers of these LAMP variants were designed based on the same target sequence as the DAMP method. As shown in Figure S15, all three LAMP variants showed stronger nonspecific amplification signals with rising background curves compared to the DAMP method. Further, we examined whether extended reaction time could lead to false positive signals caused by nonspecific exponential amplification. As shown in Figure S16A, false-positive signals were not observed for the NTC in the DAMP assay even after 120-min incubation but present in six-primer LAMP's NTC (Figure S16B). As a result, the DAMP assay provided ultralow nonspecific amplification and enabled highly sensitive and specific nucleic acid detection.

\section{Analytical performance of the DAMP assay}

To determine the sensitivity of the DAMP assay, we detected different plasmid HIV-1 DNA targets ranging from $10^{1}$ to $10^{7}$ copies $/ \mu \mathrm{L}$. As shown in Figure 6A, our DAMP assay could detect 100 copies HIV DNA per reaction, which is equal to or better than that of the sixprimer LAMP (Figure S17A and S18A) and the real-time PCR method (Figure S17B). Furthermore, due to higher amplification efficiency, the DAMP assay showed shorter threshold time compared to that of LAMP assay (Figure 6B and S19A). In addition, to demonstrate the feasibility of our DAMP assay for RNA detection, we detected the HIV-1 RNA using reverse transcription DAMP (RT-DAMP). HIV-1 RNA was extracted from inactivated HIV virus in plasma (AcroMetrix ${ }^{\mathrm{TM}}$ HIV-1 High Control, Thermo Fisher Scientific). As shown in Figure 6C and S20, RT-DAMP was able to consistently detect 350 copies for HIV-1 RNA target with ultralow specificity and faster amplification, compared to six-primer RT-LAMP (Figure 6D). 
To evaluate the versatility of our DAMP assay, we applied our DAMP method to detect $E$. coli $\mathrm{B}$ ma/B gene and compared its performance with the previously published six-primer LAMP assay ${ }^{16}$. As shown in Figure 6E, DAMP assay could achieve a sensitivity of 100 copies per reaction, which was comparable to those of LAMP assay (Figure S17C) and the real-time PCR method (Figure S17D). However, compared to the LAMP assay, DAMP showed faster amplification speed (Figure 6F and S19B). Similar to the above-mentioned HIV DNA detection, the DAMP assay for $E$. coli DNA had ultralow background fluorescence signals and did not show false-positive results after 120-min incubation (Figure S21). Collectively, all these data showed that DAMP assay was a rapid, versatile, sensitive isothermal amplification method with ultralow nonspecific signal.

\section{CONCLUSION}

In this study, we developed a DAMP (RT-DAMP) method for highly sensitive and specific nucleic acid isothermal amplification. Our method takes advantage of the "dual-priming" extension mechanism to enable highly reliable and specific molecular detection. Compared to the conventional LAMP assay, our DAMP method offers several advantages: i) ultralow nonspecific signal. DAMP showed an ultralow nonspecific signal even after two-hour incubation due to the unique inner primer design and the addition of two pairing-competition primers. ii) Shorter amplification time. The DAMP assay had higher amplification efficiency with shorter detection time because of its "dual-priming" strand extension mechanism which could simultaneously generate multiple basic structures for cycling amplification. iii) Simpler primer design. Unlike conventional six-primer LAMP, the six-primers of our DAMP assay only recognized six sites, which simplified the complexity of primer design and reduced the potential risk of forming false-positive. Also, we provide a free online software (https://github.com/xuzhiheng001/DAMP-Design) to facilitate the primer design. In addition, our DAMP assay was shown to be versatile for detection of various nucleic acid targets. Therefore, the DAMP assay described here has great potential for infectious disease diagnostics, food safety monitoring, and cancer early screening as a point of care testing, particularly in resource-limited clinical settings.

\section{Supplementary Material}

Refer to Web version on PubMed Central for supplementary material.

\section{ACKNOWLEDGMENT}

The work was supported, in part, by NIH Grants R01EB023607, R01CA214072, and R21TW010625.

\section{REFERENCES}

(1). Schachter J; Moncada J; Liska S; Shayevich C; Klausner JD Sex. Transm. Dis. 2008, 35, 637-642. [PubMed: 18520976]

(2). Rodriguez-Lazaro D; Lombard B; Smith H; Rzezutka A; D’Agostino M; Helmuth R; Schroeter A; Malorny B; Miko A; Guerra B Trends Food Sci. Technol. 2007, 18, 306-319.

(3). Pai M; Flores LL; Pai N; Hubbard A; Riley LW; Colford JM Jr The Lancet infectious diseases 2003, 3, 633-643. [PubMed: 14522262]

(4). Monis PT; Giglio S Infect. Genet. Evol. 2006, 6, 2-12. [PubMed: 16169776] 
(5). Zhao Y; Chen F; Li Q; Wang L; Fan C Chem. Rev. 2015, 115, 12491-12545. [PubMed: 26551336]

(6). Notomi T; Okayama H; Masubuchi H; Yonekawa T; Watanabe K; Amino N; Hase T Nucleic Acids Res. 2000, 28, e63-e63. [PubMed: 10871386]

(7). Gabrielle M; Schukkink RA; van Gemen B Microbiology 1993, 139, 2423-2429.

(8). Piepenburg O; Williams CH; Stemple DL; Armes NA PLoS Biol. 2006, 4, e204. [PubMed: 16756388]

(9). Vincent M; Xu Y; Kong H EMBO Rep. 2004, 5, 795-800. [PubMed: 15247927]

(10). Zhang X; Lowe SB; Gooding JJ Biosens. Bioelectron. 2014, 61, 491-499. [PubMed: 24949822]

(11). Mori Y; Notomi TJ Infect. Chemother. 2009, 15, 62-69.

(12). Nagamine K; Hase T; Notomi T Mol. Cell. Probes 2002, 16, 223-229. [PubMed: 12144774]

(13). Gandelman O; Jackson R; Kiddle G; Tisi L Int. J. Mol. Sci. 2011, 12, 9108-9124. [PubMed: 22272122]

(14). Martineau RL; Murray SA; Ci S; Gao W; Chao S.-h.; Meldrum DR. Anal. Chem. 2016, 89, 625632. [PubMed: 27809497]

(15). Eiken Chemical Co., L. https://primerexplorer.jp/e/v4manual/pdf/ PrimerExplorerV4_Manual_1.pdf

(16). Hill J; Beriwal S; Chandra I; Paul VK; Kapil A; Singh T; Wadowsky RM; Singh V; Goyal A; Jahnukainen TJ Clin. Microbiol. 2008, 46, 2800-2804.

(17). Kimura Y; de Hoon MJ; Aoki S; Ishizu Y; Kawai Y; Kogo Y; Daub CO; Lezhava A; Arner E; Hayashizaki Y Nucleic Acids Res. 2011, 39, e59-e59. [PubMed: 21310714]

(18). Tomita N; Mori Y; Kanda H; Notomi T Nat. Protoc. 2008, 3, 877. [PubMed: 18451795]

(19). Rees WA; Yager TD; Korte J; Von Hippel PH Biochemistry 1993, 32, 137-144. [PubMed: 8418834]

(20). Mok E; Wee E; Wang Y; Trau M Sci. Rep. 2016, 6, 37837. [PubMed: 27910874]

(21). Rajendrakumar CS; Suryanarayana T; Reddy AR FEBS Lett. 1997, 410, 201-205. [PubMed: 9237629]

(22). Yang L; Arora K; Beard WA; Wilson SH; Schlick TJ Am. Chem. Soc. 2004, 126, 8441-8453.

(23). Liao S-C; Peng J; Mauk MG; Awasthi S; Song J; Friedman H; Bau HH; Liu C Sensor Actuat. BChem. 2016, 229, 232-238.

(24). Kadimisetty K; Song J; Doto AM; Hwang Y; Peng J; Mauk MG; Bushman FD; Gross R; Jarvis JN; Liu C Biosens. Bioelectron. 2018, 109, 156-163. [PubMed: 29550739] 
A

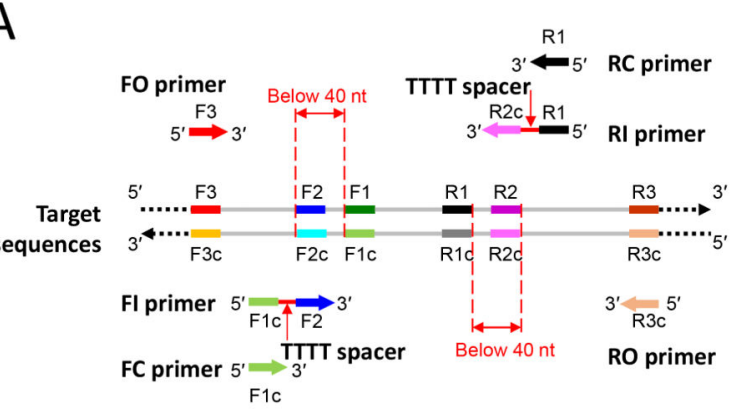

C

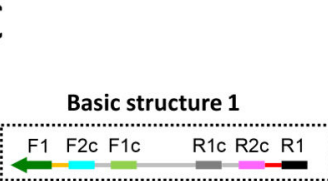

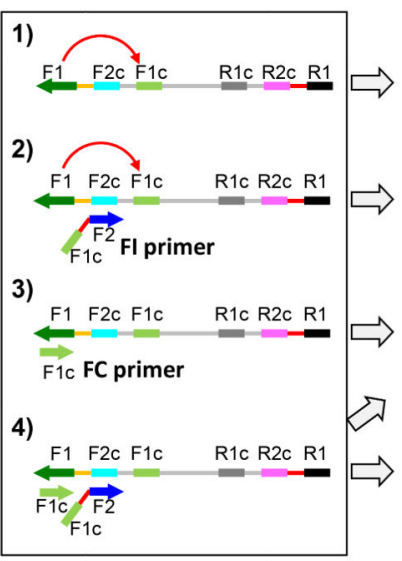

B
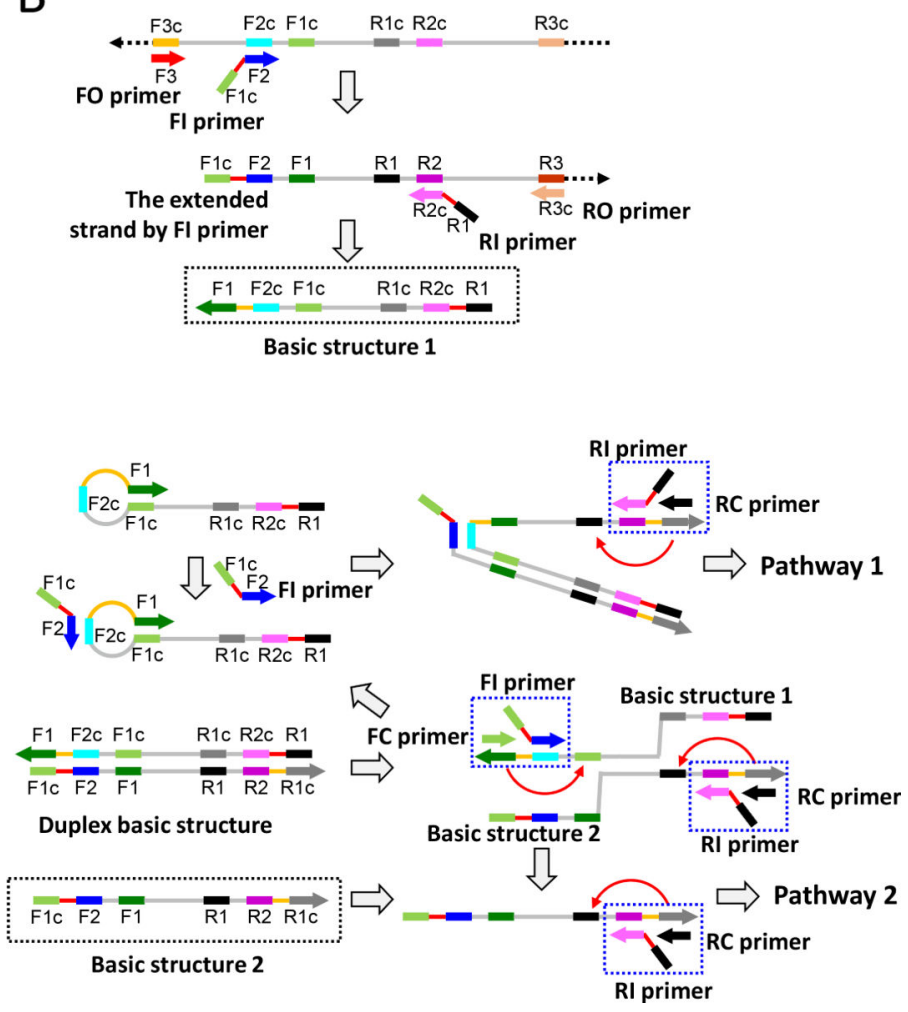

Figure 1.

Principle of the DAMP assay: (A) Primer design of the DAMP method. FO, forward outer primer; RO, reverse outer primer; FI, forward inner primer; RI, reverse inner primer; FC, forward pairing-competition primer; and $\mathrm{RC}$, reverse pairing-competition primer. (B) Basic structure producing step. (C) Cycling amplification step. More details of pathway 1 and 2 are given in Figure S3. 


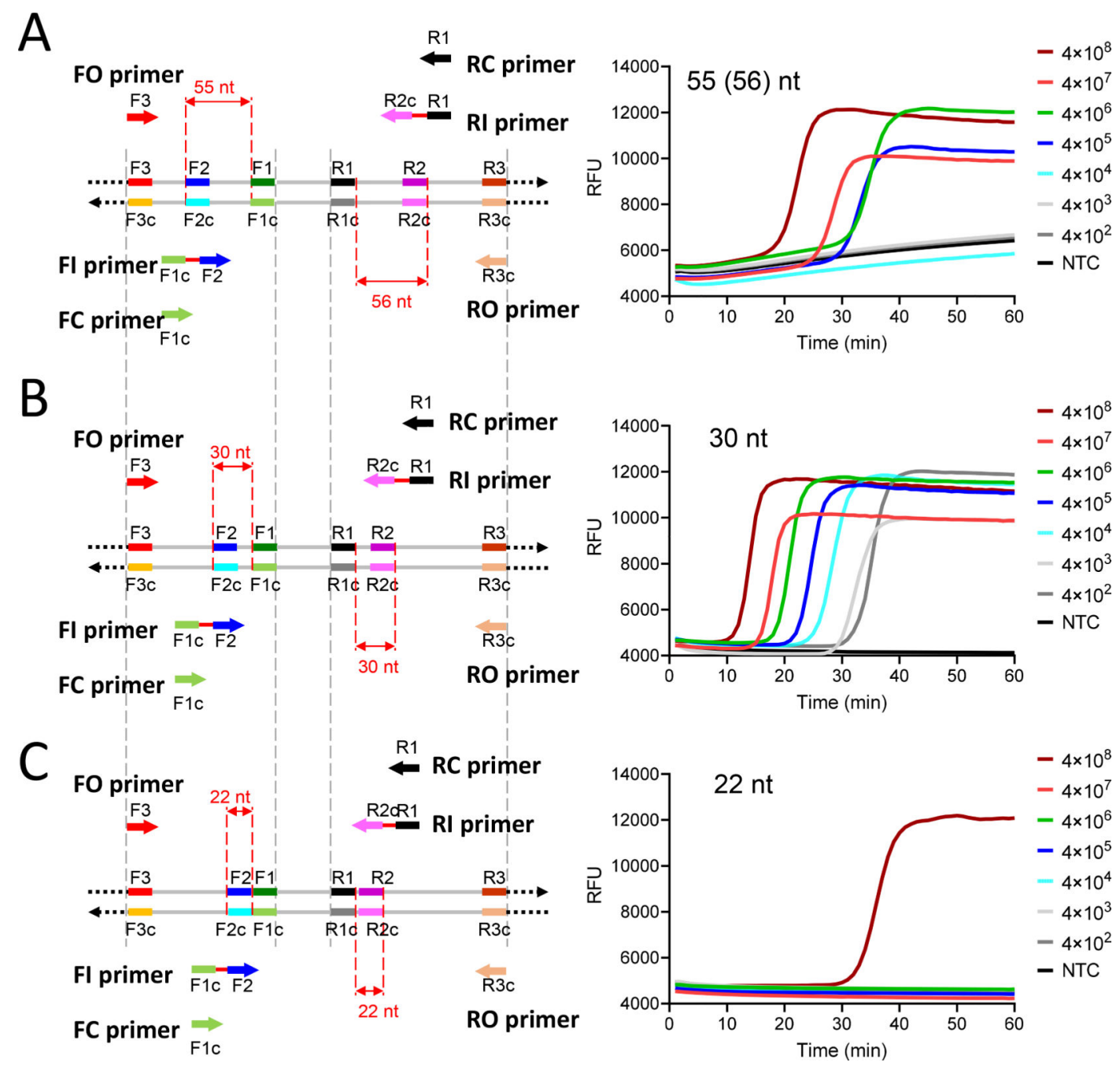

Figure 2.

Effect of the distances of two target sites used for inner primer design on DAMP amplification efficiency: (A) Design of the inner primers with a distance of 55 (56) nt (Left) and its real-time fluorescence DAMP detection (Right). (B) Design of the inner primers with a distance of $30 \mathrm{nt}$ (Left) and its real-time fluorescence DAMP detection (Right). (C) Design of the inner primers with a distance of $22 \mathrm{nt}$ (Left) and its real-time fluorescence DAMP detection (Right). The vertical gray dash lines denote the same amplification region in the target sequences. 10-fold serial dilution of HIV-1 DNA targets (from $4 \times 10^{8}$ to $4 \times 10^{2}$ copies) were used. 


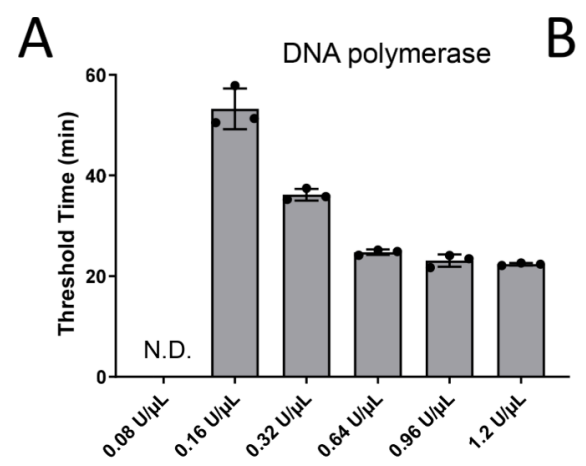

B dNTPs

C

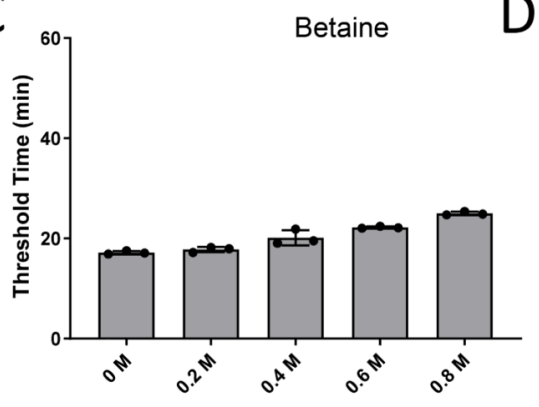

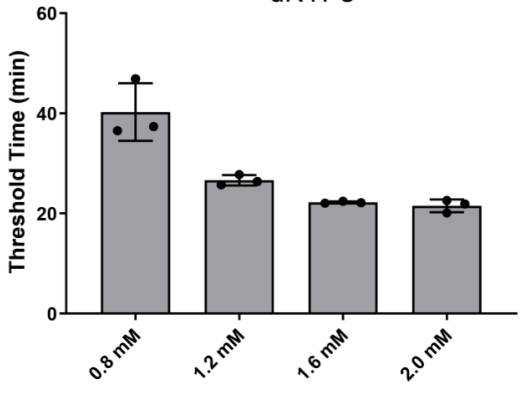

$\mathrm{MgSO}_{4}$

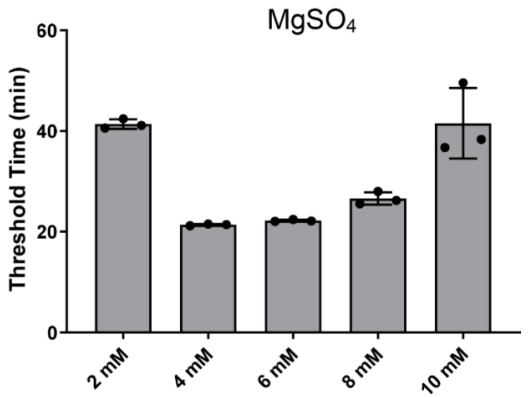

Figure 3.

Effect of the concentration of different reaction components on DAMP assay for HIV-1 DNA detection: (A) DNA polymerase, (B) dNTPs, (C) betaine, and (D) $\mathrm{MgSO}_{4} \cdot 10^{5}$ copies HIV-1 p24 gene cDNA was used as the target sequence. Error bars represent the standard deviations at three replicates $(n=3)$. N.D., not detectable within 60-min DAMP reaction. 

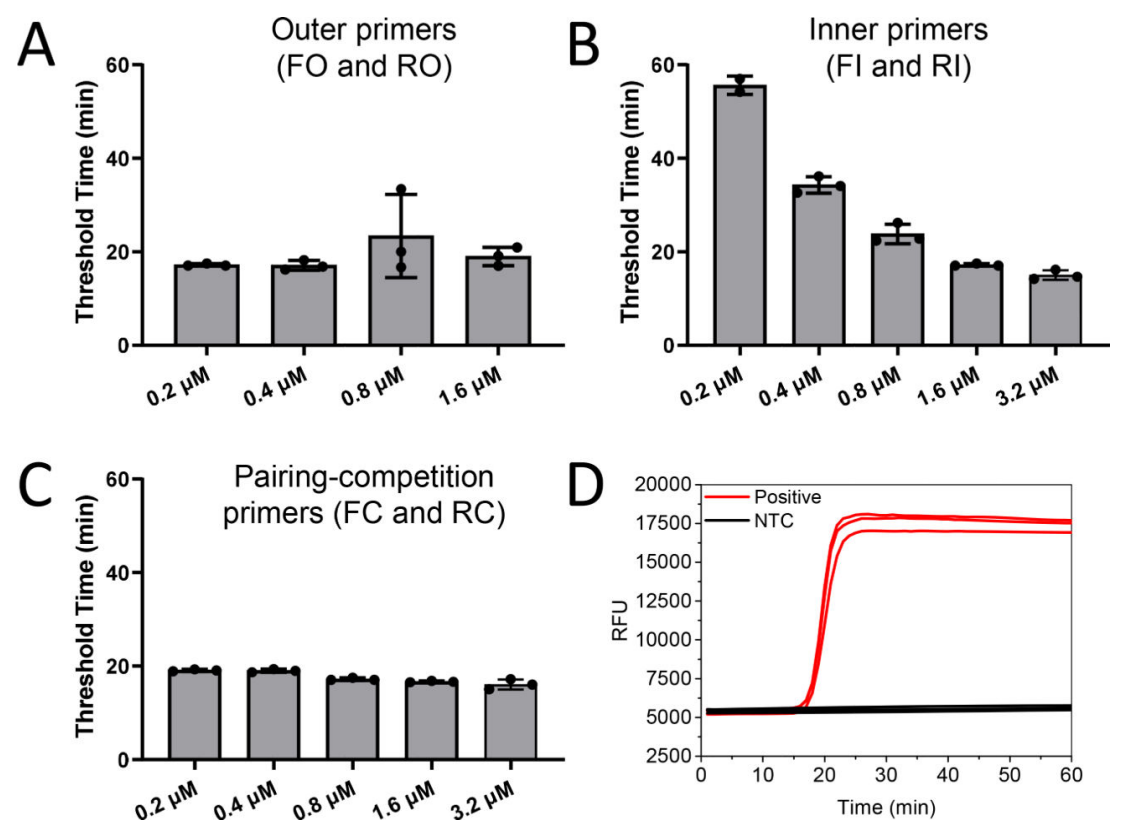

Figure 4.

Optimization of the DAMP primers concentrations: (A) FO and RO, (B) FI and RI, and (C) FC and RC. $10^{5}$ copies HIV-1 cDNA templates were used as template in the DAMP assay. Error bars represent the standard deviations at three replicates $(n=3)$. (D) Real-time fluorescence DAMP assay of HIV DNA using the optimal primer concentration. Positive, three replicated reactions with $10^{5}$ copies HIV-1 p24 gene cDNA templates. NTC, three replicated non-template control reactions. 
A

Six-primer LAMP
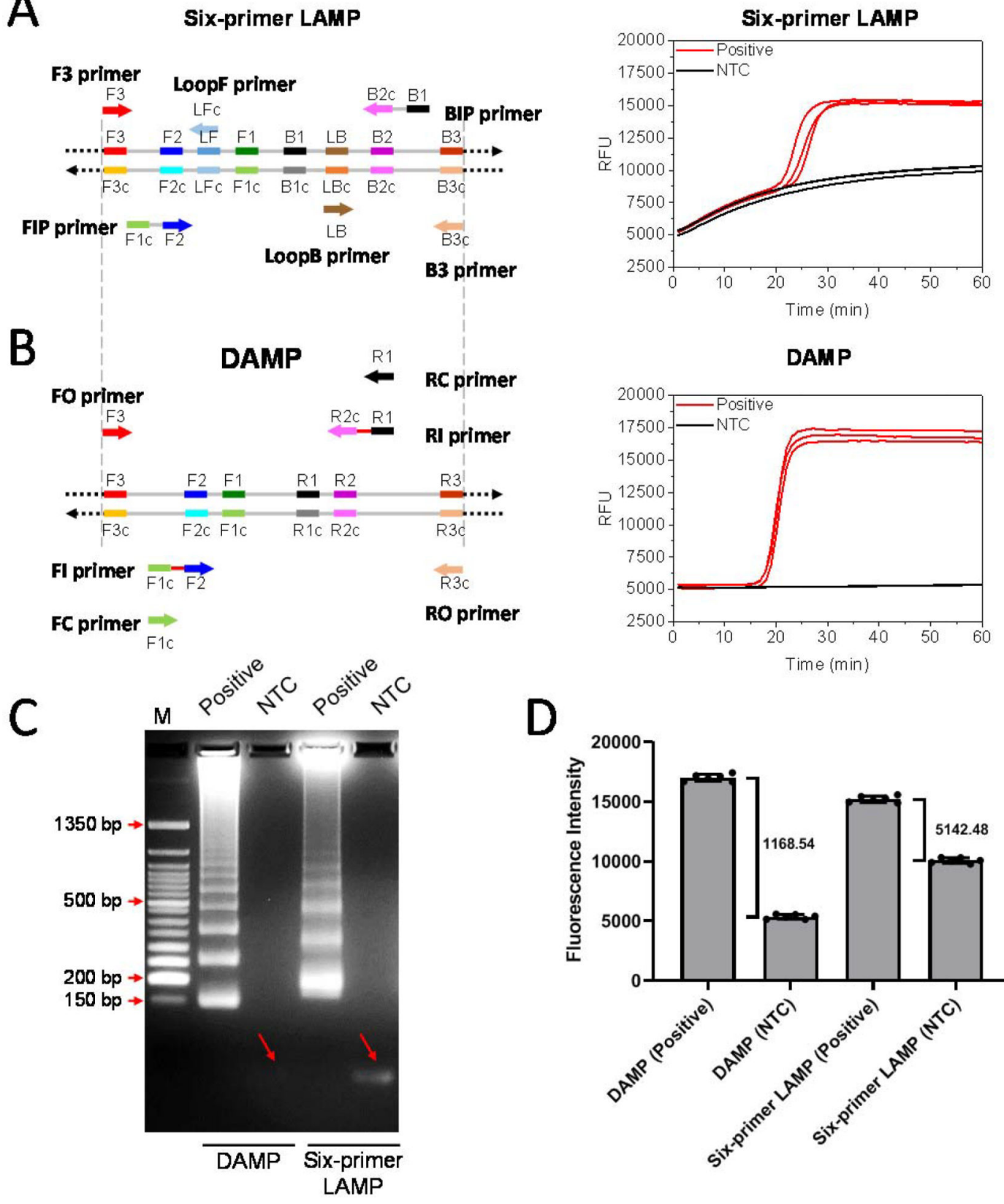

D

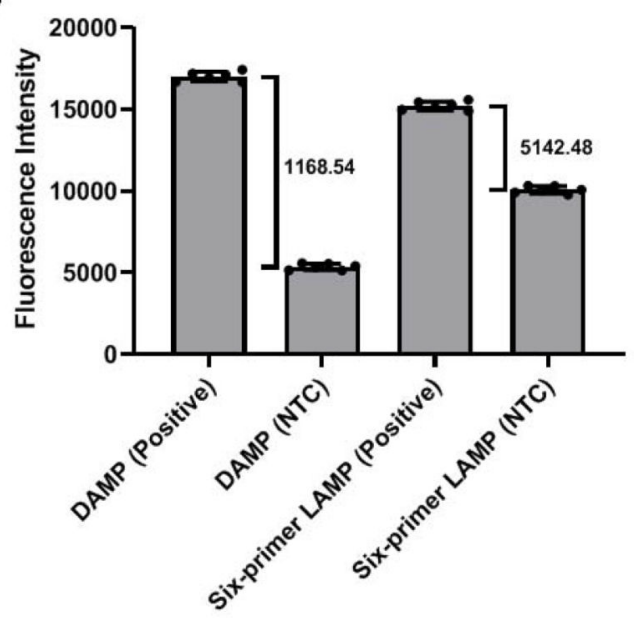

Figure 5.

Comparison of the six-primer LAMP and DAMP assay for the detection of HIV-1 DNA by targeting the same DNA sequence. (A) Primer design of six-primer LAMP (Left) and its real-time fluorescence LAMP detection (Right). (B) Primer design of DAMP (Left) and its real-time fluorescence DAMP detection (Right). (C) Electropherograms of DAMP and sixprimer LAMP products in a 3\% agarose gel. (D) Comparison of endpoint fluorescence intensity of the DAMP products and six-primer LAMP products after 60-min reactions. Error bars represent the standard deviations at six replicates $(n=6)$. Positive, $10^{5}$ copies HIV-1 p24 gene cDNA templates. NTC, three replicated non-template control reactions. 

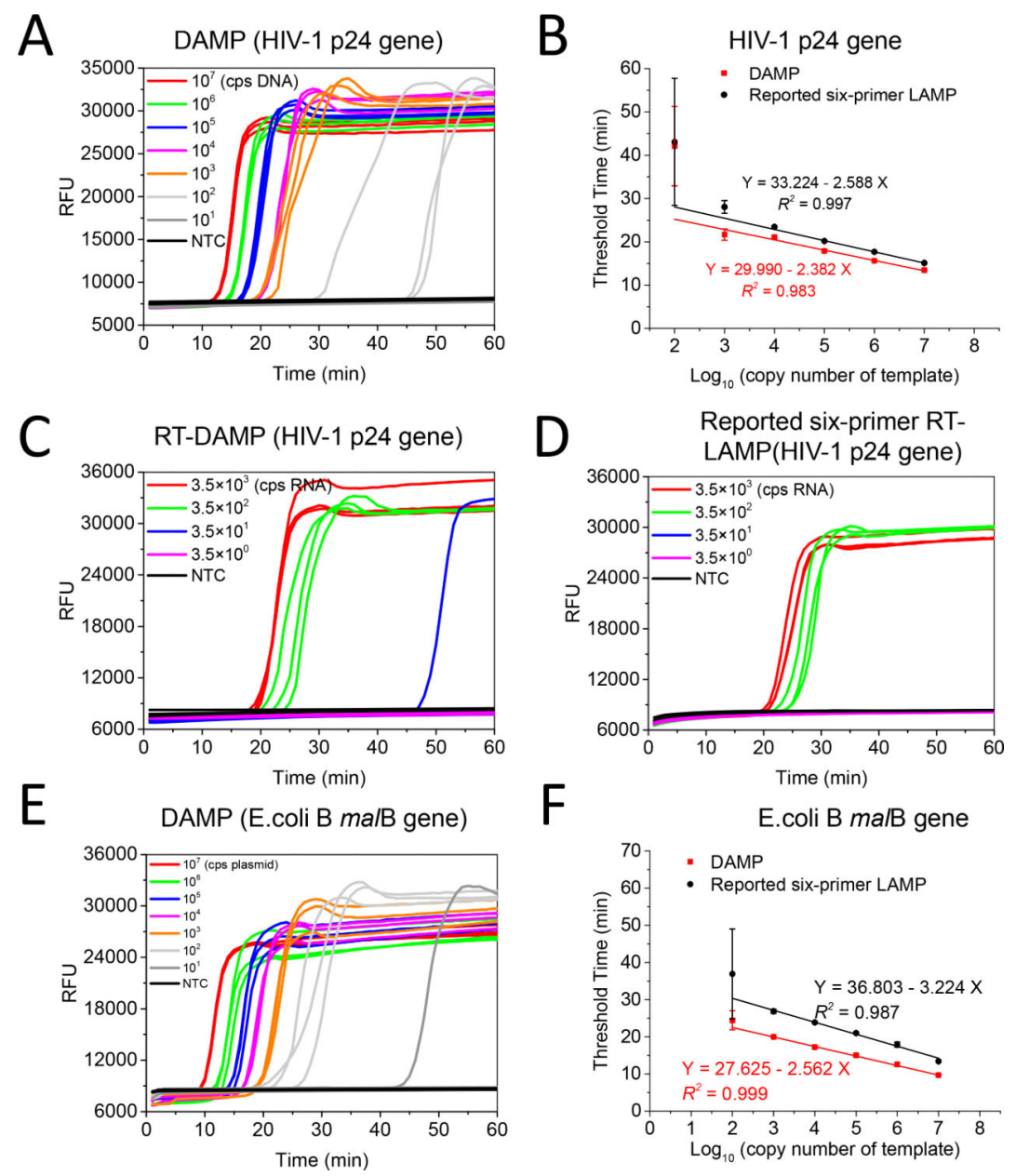

Figure 6.

Comparison of the DAMP (RT-DAMP) and six-primer LAMP (RT-LAMP) assays for HIV-1 DNA (RNA) detection and versatility evaluation of our DAMP assay by detecting $E$. coli DNA. (A) Sensitivity of DAMP assay for the detection of HIV-1 p24 gene cDNA sequence. (B) Threshold time and its linear relationship with the $\log _{10}$ of copy number of HIV DNA templates for DAMP and six-primer LAMP. (C) Sensitivity of RT-DAMP for the detection of HIV-1 genomic RNA extracted from HIV-1 plasma control samples. (D) Sensitivity of RT-LAMP for the detection of HIV-1 genomic RNA extracted from HIV-1 plasma control samples. (E) Sensitivity of DAMP to amplify the $E$. coli $B$ malB gene sequence. $(\mathrm{F})$ The linear relationship between threshold time and the $\log _{10}$ of copy number of $E$. coli DNA templates by using our DAMP assay and the previously reported six-primer LAMP. Cps, copy number. Three replicates were set up for every reaction. NTC, three replicated nontemplate control reactions. Error bars represent the standard deviations of threshold times at three replicates $(n=3)$. 